This item was submitted to Loughborough's Research Repository by the author.

Items in Figshare are protected by copyright, with all rights reserved, unless otherwise indicated.

\title{
An atomic finite element model for biodegradable polymers. Part 2. A model for change in Young's modulus due to polymer chain scission
}

PLEASE CITE THE PUBLISHED VERSION

https://doi.org/10.1016/j.jmbbm.2015.07.010

\section{PUBLISHER}

(C) Elsevier

\section{VERSION}

AM (Accepted Manuscript)

\section{PUBLISHER STATEMENT}

This work is made available according to the conditions of the Creative Commons Attribution-NonCommercialNoDerivatives 4.0 International (CC BY-NC-ND 4.0) licence. Full details of this licence are available at: https://creativecommons.org/licenses/by-nc-nd/4.0/

\section{LICENCE}

CC BY-NC-ND 4.0

\section{REPOSITORY RECORD}

Gleadall, Andy, Jingzhe Pan, and Marc-Anton Kruft. 2015. "An Atomic Finite Element Model for Biodegradable Polymers. Part 2. A Model for Change in Young's Modulus Due to Polymer Chain Scission”. Loughborough University. https://hdl.handle.net/2134/26779. 


\title{
An atomic finite element model for biodegradable polymers. Part 2. A model for change in Young's modulus due to polymer chain scission
}

\author{
Andrew Gleadall*a ${ }^{*}$ Jingzhe $\mathrm{Pan}^{\mathrm{b}}$ and Marc-Anton $\mathrm{Kruft}^{\mathrm{c}}$ \\ ${ }^{a}$ Manufacturing and Process Technologies, Faculty of Engineering, University of Nottingham, \\ University Park, Nottingham, NG7 2RD, UK \\ ${ }^{\mathrm{b}}$ Department of Engineering, University of Leicester, Leicester, LE1 7RH, UK \\ ${ }^{c}$ Corbion Purac Biomaterials, P.O. Box 21, 4200 AA Gorinchem, The Netherlands \\ * Corresponding author. Tel: +44(0)115 823 2507; Fax: +44(0)115 951 3800; E-mail: \\ andrew.gleadall@nottingham.ac.uk;
}

Final publication is available at Elsevier via https://doi.org/10.1016/j.jmbbm.2015.07.010

\begin{abstract}
Atomic simulations were undertaken to analyse the effect of polymer chain scission on amorphous poly(lactide) during degradation. Many experimental studies have analysed mechanical properties degradation but relatively few computation studies have been conducted. Such studies are valuable for supporting the design of bioresorbable medical devices. Hence in this paper, an Effective Cavity Theory for the degradation of Young's modulus was developed. Atomic simulations indicated that a volume of reduced-stiffness polymer may exist around chain scissions. In the Effective Cavity Theory, each chain scission is considered to instantiate an effective cavity. Finite Element Analysis simulations were conducted to model the effect of the cavities on Young's modulus. Since polymer crystallinity affects mechanical properties, the effect of increases in crystallinity during degradation on Young's modulus is also considered. To demonstrate the ability of the Effective Cavity Theory, it was fitted to several sets of experimental data for Young's modulus in the literature.
\end{abstract}

Key words: Biodegradable polymers, degradation, Young's modulus, mechanical properties, computer modelling.

\section{Introduction}

Biodegradable polymers such as poly(lactide) are used in medical devices that help support broken bones during the healing process. After a period of months or years, the devices 
degrade and are absorbed by the body. The mechanical properties during degradation are of interest. Young's modulus of biodegradable polymer is related to many factors of which molecular weight and crystallinity are particularly important. During degradation, crystallinity generally increases while molecular weight decreases. The increase in crystallinity may cause Young's modulus to increase (Tsuji and Ikada, 1995) while molecular weight reduction results in a Young's modulus decrease. Theoretical models help improve understanding of how mechanical properties degrade and can be used to simulate degradation in order to design the optimal device. Wang etc al. (Wang et al., 2010) considered the entropy spring model for rubbery amorphous polymer and considers short polymer chains to have negligible effect on the entropy change in a linear biodegradable polymer during its deformation. The model was demonstrated to be able to fit experimental data for Young's modulus degradation. However, many biodegradable polymers are used below their glass transition temperature, in which case there is no current theoretical model that can be used to describe the degradation of Young's modulus. The effect of entropy reduction on mechanical properties is not expected to be a major factors for polymers used below their glass transition temperature. Numerical analysis of the effect of polymer chain scission on Young's modulus both above and below the glass transition temperature was carried out by Ding et al. (Ding et al., 2011) using molecular dynamics (MD) simulations. It was found that below the glass transition temperature, chain scission reduces Young's modulus as a result of lowering the Lennard-Jones interaction between polymer chains. The MD simulations in that study considered polyethylene chains. The analysis of complex biodegradable polymer molecules such as poly(lactide) was not possible due to computational demands.

This paper presents a study on the effect of polymer chain scission on Young's modulus of biodegradable polymers. The atomic finite element method (AFEM) is used, which was presented in the first of paper in this two-part series (Gleadall et al.). AFEM simulations are much less computationally demanding than MD so can be used for amorphous poly(lactide) polymer structures. A large number of scissions are applied to understand their effect on Young's modulus. Also, individual chain scissions are analysed the atomic-scale in order to understand the local effects of chain scission on force transfer through the polymer. Based on these new understandings obtained using the atomistic simulations, an Effective Cavity Theory is proposed, which can simply model the degradation of Young's modulus due to chain scission. The effect of increasing crystallinity is also considered in the model. 


\section{The atomic finite element method (AFEM)}

The atomic finite element model (AFEM) model has been described in detail in the accompanying paper (Gleadall et al.). Interatomic potential energy functions for covalent bonds and nonbonded interactions, which are taken from the PLAFF2 molecular dynamics force field for poly(lactide) (McAliley, 2009), are used in atomic finite element analysis simulations. The nonlinear interatomic potential energy functions are adapted to enable static linear analysis. The AFEM simulation technique is therefore greatly more computationally efficient than molecular dynamics (MD) simulations. This allows the analysis of polymer chain scission in large amorphous polymer structures. In AFEM, there a four main types of elements: three are used to simulate covalent bonds while the fourth represents nonbonded interactions including Lennard-Jones and Coulomb interactions. The three covalent bond elements are referred to as bond-stretch, bond-angle and bond-dihedral elements. They model the covalent bonds' stiffness with respect to interatomic separation of two covalently bonded atoms, the angle between two covalent bonds, and the rotation of a polymer chain about a covalent bond, respectively. During the AFEM simulations, a strain is applied to the atomic structure and the resulting interatomic potential energy increase in the AFEM simulations, which indicates stain energy, is used to identify Young's modulus for the polymer. Since the simulations are for linear analysis, the magnitude of applied strain does not affect the calculation of Young's modulus. A value of $2 \%$ strain is used. A nonbond cutoff distance of $0.95 \mathrm{~nm}$ is utilised unless otherwise indicated since this value is often implemented in MD simulations. The atomic structure used in this study is the amorphous unit cell for poly(lactide), which was also used in the accompanying paper (Gleadall et al.). The structure was obtained from the PhD thesis by McAliley (McAliley, 2009). Scissions are applied in the AFEM simulations by removing one polymer repeat unit from the structure.

For an atomic structure subject to tensile strain the unit cell images on opposite sides of the central unit cell apply opposite forces to the central unit cell in order to elongate it. These forces are transferred through AFEM elements in the structure from one side to the other. Fig. 1 (a) shows how equal and opposite forces applied to atoms 1 and 3 in the figure must transfer through the two elements and through the central atom. The labelled forces in the figure are the forces that must be applied to the atoms (by other AFEM elements or external forces) in order to strain the elements being considered. The force that is transferred through an element can be calculated based on the stiffness of the element and the atomic displacement. For 3D simulations, the component of the force that is in the direction of applied stress is of interest. Since an atom may be utilised in several elements, the force that transfers through the atom is calculated as the sum of the force contributions from all the 
elements in which the considered atom is contained. The bond-angle and bond-dihedral elements contain more than two atoms, and the force that is transferred through the element changes at different positions within the element. In order to determine the force transferred through a particular atom, an imaginary cut is applied to the structure immediately next to the atom in question. This is shown in Fig. 1 (b) with respect to atom 1 for a bond-angle element connecting three atoms. The force that is transferred through the element at the location of the imaginary cut plane in the figure is calculated as the sum of forces (applied to the element by other elements) for all atoms that are in the positive direction of applied strain (atoms 2 and 3 in the figure).

a)
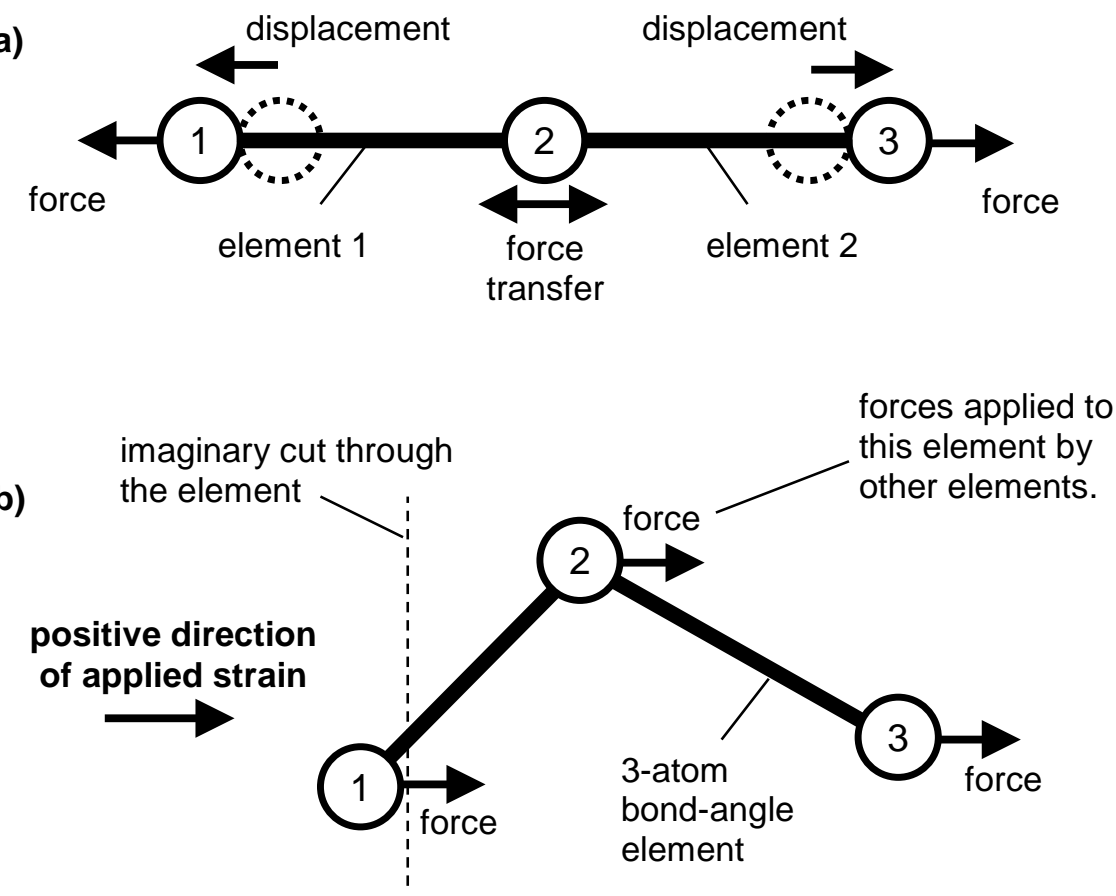

Fig. 1 a) A simple transfer of force through two bond-stretch elements and b) the force transferred to atom 1 by a bond-angle element between three atoms can be considered using an imaginary cut through the element.

\section{Atomic simulations for polymer chain scission}

During degradation the value of Young's modulus decreases as a result of polymer chain scission and a reduction in molecular weight. The AFEM simulations can be used to simulate the degradation of Young's modulus and help understand the trends that are found experimentally. For the purpose of analysing the effect of chain scission on Young's modulus, 
chain scissions are applied cumulatively the amorphous structure and Young's modulus is calculated at various molecular weights. Chain scission of the crystalline phase in semicrystalline poly(lactide) is not expected to play an important role in degradation since the crystalline phase degrades slowly compared to the amorphous phase. Therefore a relationship between Young's modulus and molecular weight is only studied for the amorphous phase. Fig. 2 (a) shows the relationship between normalised Young's modulus and molecular weight that is found in the AFEM simulations for the amorphous polymer unit cell. The values of Young's modulus calculated are normalised by the values found at 8950 $\mathrm{g} \mathrm{mol}^{-1}$ to ensure clarity in the figure. This is the number averaged molecular weight after three scissions have been applied to each of the original polymer chains. The results for a regular AFEM setup are indicated by the solid line and demonstrate a reduction in Young's modulus only after the molecular weight has considerably reduced. This trend that Young's modulus degradation is initially delayed behind molecular weight degradation is also found in many experimental studies in the literature (Lam et al., 1994; Migliaresi et al., 1994; Saha and Tsuji, 2006; Tsuji, 2000, 2002, 2003; Tsuji and Muramatsu, 2001; Tsuji and Suzuyoshi, 2002a, b; Weir et al., 2004; Yuan et al., 2002). To test the sensitivity of the AFEM technique, the AFEM setup is varied in two ways: (i) the stiffness of nonbond AFEM elements are reduced by $99 \%$, and (ii) the nonbond cut-off distance is reduced from $0.95 \mathrm{~nm}$ to $0.5 \mathrm{~nm}$. The scissions are applied to the same polymer repeat units in all three test setups. In both of the modified setups, the Young's modulus degradation occurs earlier, with respect to molecular weight degradation, than for the regular AFEM setup. This is due to the fact that both variations result in an increased contribution to overall Young's modulus from the covalent bond elements of the main polymer chains. When the polymer chains have a more important contribution, the scissions applied to these chains have a greater impact on Young's modulus. 
a)

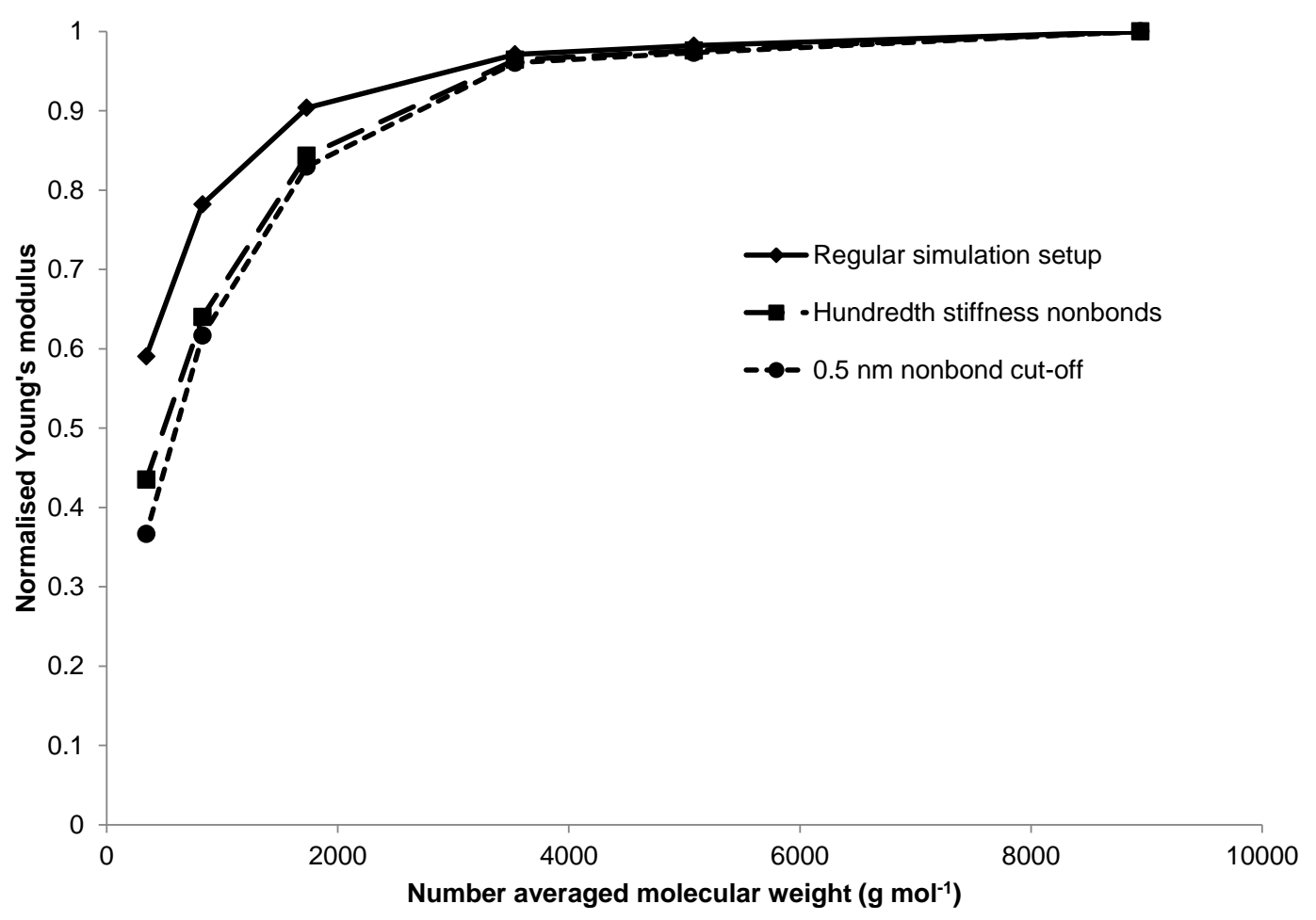

b)

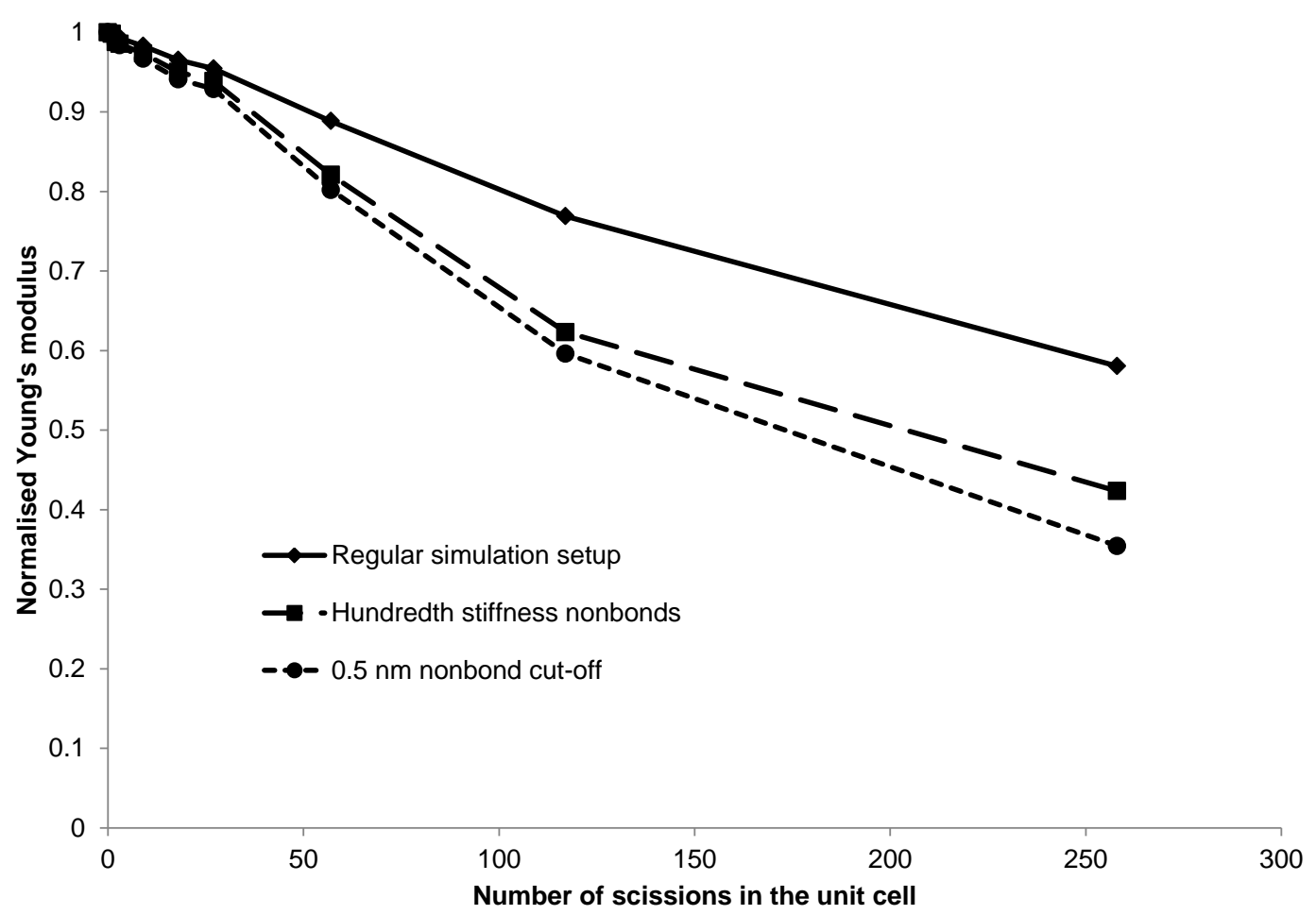

Fig. 2 AFEM simulations are used to find a relationship for normalised Young's modulus versus a) number averaged molecular weight and b) the number of chain scissions in the amorphous polymer unit cell. Simulations are conducted with a regular AFEM setup, with 99\% reduced stiffness nonbonds, and with a reduced nonbond cut-off of $0.5 \mathrm{~nm}$. 
Fig. 2 (b) shows the relationship between normalised Young's modulus and the number of chains scissions applied to the amorphous polymer unit cell. It can be seen to be almost linear for all three AFEM setups. This can explain why Young's modulus drops more suddenly at lower molecular weights. Consider the chain scission of a polymer structure containing 10 initial polymer chains. Table 1 indicates the number of scissions that are required to reduce normalised molecular weight by various amounts. To reduce the normalised molecular weight by $\approx 0.1$ takes just 1 scission initially. After 40 scissions have been applied such that normalised molecular weight is 0.2 however, 50 scissions are required in order to reduce the normalised molecular weight by 0.1 . In a degraded polymer with lower molecular weight, many more scissions are required to further reduce molecular weight. Therefore for a near-linear relationship between Young's modulus and the number of scissions, as identified in Fig. 2 (b), the reduction of Young's modulus accelerates greatly as molecular weight reduces.

Table 1 Demonstration of how the number of scissions must increase acceleratedly as normalised molecular weight reduces in order to further reduce normalised molecular weight.

\begin{tabular}{lll}
\hline number of chains & number of scission & normalised $M_{n}$ \\
\hline 10 & 0 & 1.000 \\
11 & 1 & 0.909 \\
12 & 2 & 0.833 \\
15 & 5 & 0.667 \\
20 & 10 & 0.500 \\
30 & 20 & 0.333 \\
40 & 30 & 0.250 \\
50 & 40 & 0.200 \\
100 & 90 & 0.100 \\
200 & 190 & 0.050 \\
\hline
\end{tabular}

\section{AFEM simulations for effective cavities due to chain scission}

In addition to the effect of chain scission on overall Young's modulus, the effect of chain scission is investigated in terms of the interatomic force that is transferred through the structure. For such analysis it is useful to consider the effects of individual chain scissions. Fig. 3 (a) shows the amorphous poly(lactide) unit cell that is used in the AFEM simulations. One chain scission has been applied to the polymer. In Fig. 3 (b) atoms are only shown if the interatomic force that they transfer in the direction of applied strain reduces by a threshold value in the simulation with the chain scission versus the simulation without the chain scission. The threshold is chosen to be a reduction in transferred force of at least $50 \%$ of the 
average force transferred for all atoms. The clusters of atoms above and below the scission may indicate that there are volumes of polymer above and below the scission that have a reduced force. The AFEM simulations do not consider the reconfiguration of the polymer as a result of chain scission, but it is reasonable to expect the end of chain ends to be more free to move than polymer units in the middle of a chain. This extra freedom may cause the local surrounding polymer have a lower Young's modulus than a region of polymer without a chain end.

a)

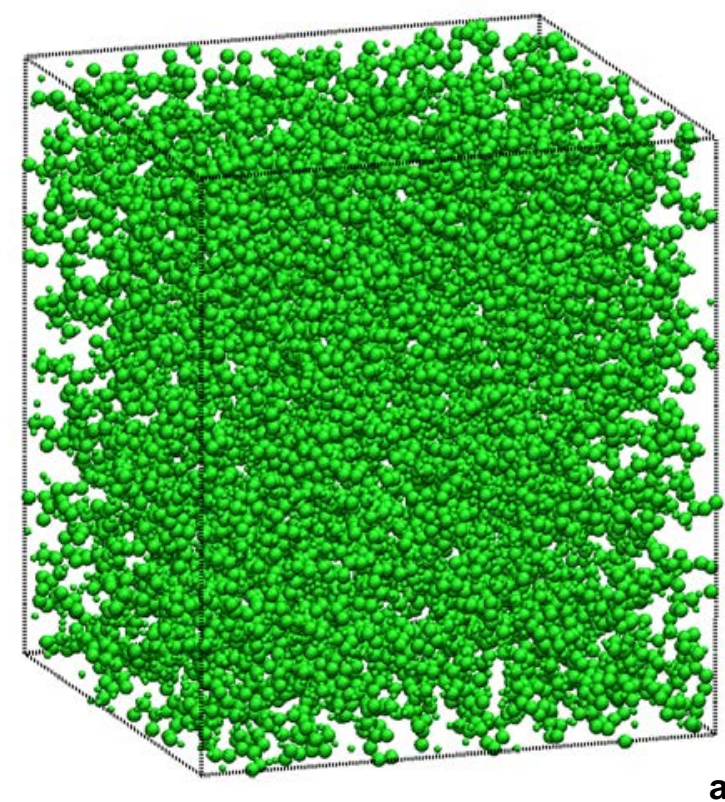

b)

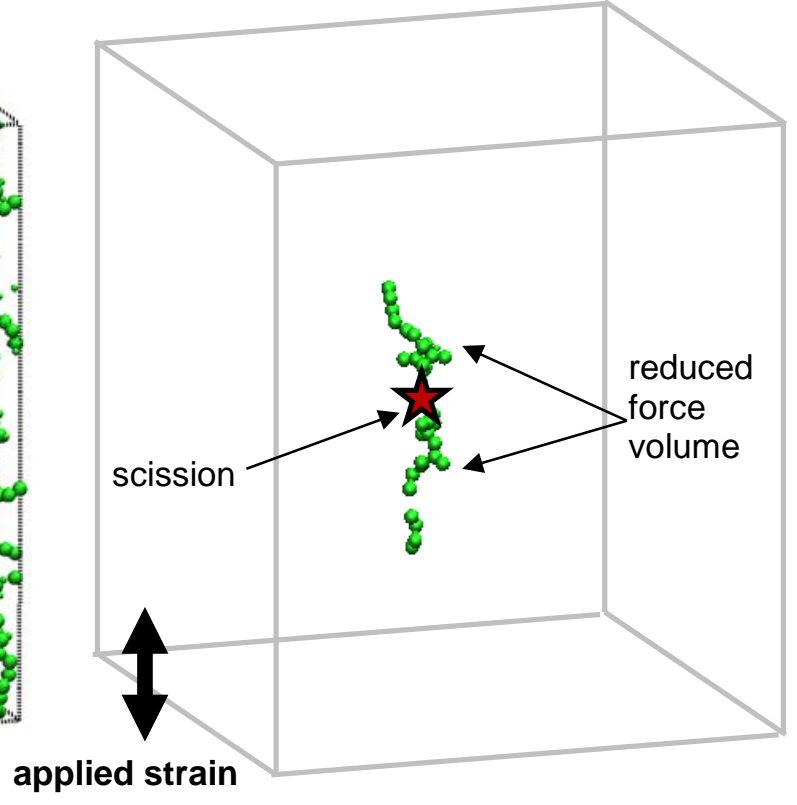

Fig. 3 The polymer unit cell is shown a) in its entirety and b) with atoms only displayed if they demonstrate a threshold reduction (as a result of the chain scission) in the force that they transfer through the polymer.

In the Effective Cavity Theory presented in this study, each scission is represented by an effective cavity. In order to identify the shape of the effective cavity, ten scissions are individually applied to random polymer units in the amorphous polymer structure used in AFEM simulations. Fig. 4 shows the AFEM results for the ten individual scissions, viewed normal to the direction of applied strain. The threshold is reduced from $50 \%$ used in Fig. 3 to $10 \%$ in Fig. 4 to enable more atoms to be visualised. The schematic in the figure demonstrates that flat-tipped cones may be used to approximate the volumes of the effective cavity that result from a chain scission. For each scission, effective cavities may be considered to exist in the shape of flat-tipped cones oriented tip-to-tip. Pairs of flat-tipped cones are overlaid onto each of the simulation results in the figure to demonstrate that such 
a shape is a suitable approximation of the reduced stiffness polymer near each chain scission. The orientation of the flat-tipped cones depends on the direction of applied strain. If the direction of applied strain changes, so too does the position of effective cavities that result from chain scission. Based on Fig. 4, a flat-tipped cone shape is used to represent the shape of effective cavities when developing the Effective Cavity Theory to relate Young's modulus to chain scission. There is some variation between the shapes of the cones in Fig. 4. The shape can be characterised (i) by the ratio of the top radius to the base radius, and (ii) by the ratio of the cone height to the base radius. The base radius, top radius and cone height are all labelled in Fig. 4. For the ten cone shapes given in Fig. 4, the ratio of the top radius to the base radius varies from approximately 0.25 to 0.5 and the ratio of the cone height to the base radius varies from approximately 1 to 1.25 . In order to account for the variation in cone dimensions during the development of the Effective Cavity Theory, a range of ratios are studied: the ratio of the top radius to the base radius is varied from 0 to 0.667 and the ratio of the cone height to the base radius is varied from 1 to 2 .

\section{Effective Cavity Theory for change in Young's modulus due to chain scissions}

The Effective Cavity Theory model for the degradation of Young's modulus in biodegradable polymers combines models for the effect of both chain scission and crystallinity on Young's modulus. In the model for chain scission, effective cavities in the shape of flat-tipped cones are considered to result from each chain scission. As the concentration of chain scissions increases in a polymer during degradation, the volume fraction of effective cavities increases. The model for chain scission effective cavities relates the volume fraction of effective cavities to Young's modulus. As crystallinity increases, Young's modulus increases because the crystallites have greater stiffness than amorphous polymer. Crystallites are generally considered to be cuboidal in shape, and hence the model for crystallinity considers a cubic crystal particle in a cubic matrix of amorphous polymer. To develop the models, the finite element analysis package COMSOL Multiphysics 4.3b (license 7074366) is used.

\subsection{Effect of chain scission on Young's modulus}

To develop the model for effective cavities associated with chain scission, one quarter of a flat tipped cone is modelled as a void in a cubic matrix, which represents amorphous polymer. The FEA model including the mesh is shown in Fig. 5. The height and large radius of the cone are set to be $1 \mathrm{~nm}$. The small radius of the cone is set to be $0.333 \mathrm{~nm}$. These 
values are adjusted and discussed later in this section to investigate the effect of cone shape on the

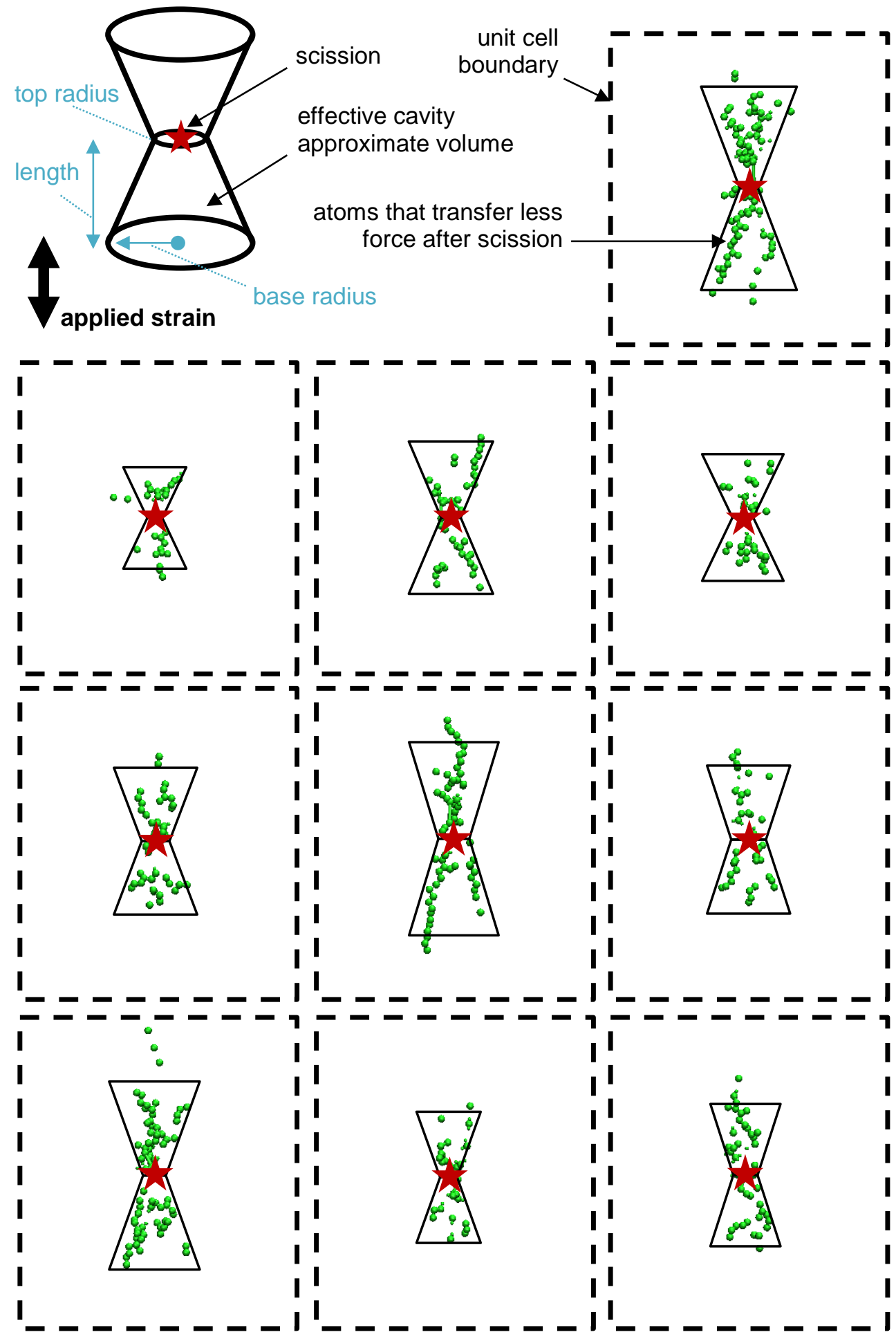

Fig. 4 Ten scissions are individually applied to the amorphous polymer. Atoms are only displayed if they demonstrate a threshold reduction in the force that they transfer through the polymer as a result of the chain scission. A volume of less stiff polymer may exist around each scission, which takes the shape of two flat-tipped cones oriented tip-to-tip in the 
direction of applied strain. This shape is chosen for the model of the Effective Cavity Theory presented in this study.

effective cavity theory. For the simulation shown in the figure, the overall cubic cell has side lengths of $1.5 \mathrm{~nm}$. Symmetry is assumed across the left, front and bottom faces of the cube in Fig. 5, which represents a cube eight times the volume containing two flat-tipped cone voids oriented tip-to-tip. Strain is applied to the top face of the cube in Fig. 5, while the back and right faces are free to displace in order to accommodate Poisson's ratio but remain planar. The bottom face is restrained in the $z$ direction. These boundary conditions represent the case that the larger cube, containing eight reflections of the cube shown in Fig. 5 , is a unit cell that repeats to infinity in all directions. During the FEA simulations that develop the Effective Cavity Theory, the size of the flat-tipped cone effective cavity is kept constant while the overall cubic cell size reduces. A smaller cubic cell represents a reduced separation between chain scissions in the polymer and therefore more scissions per unit volume and a more degraded polymer.

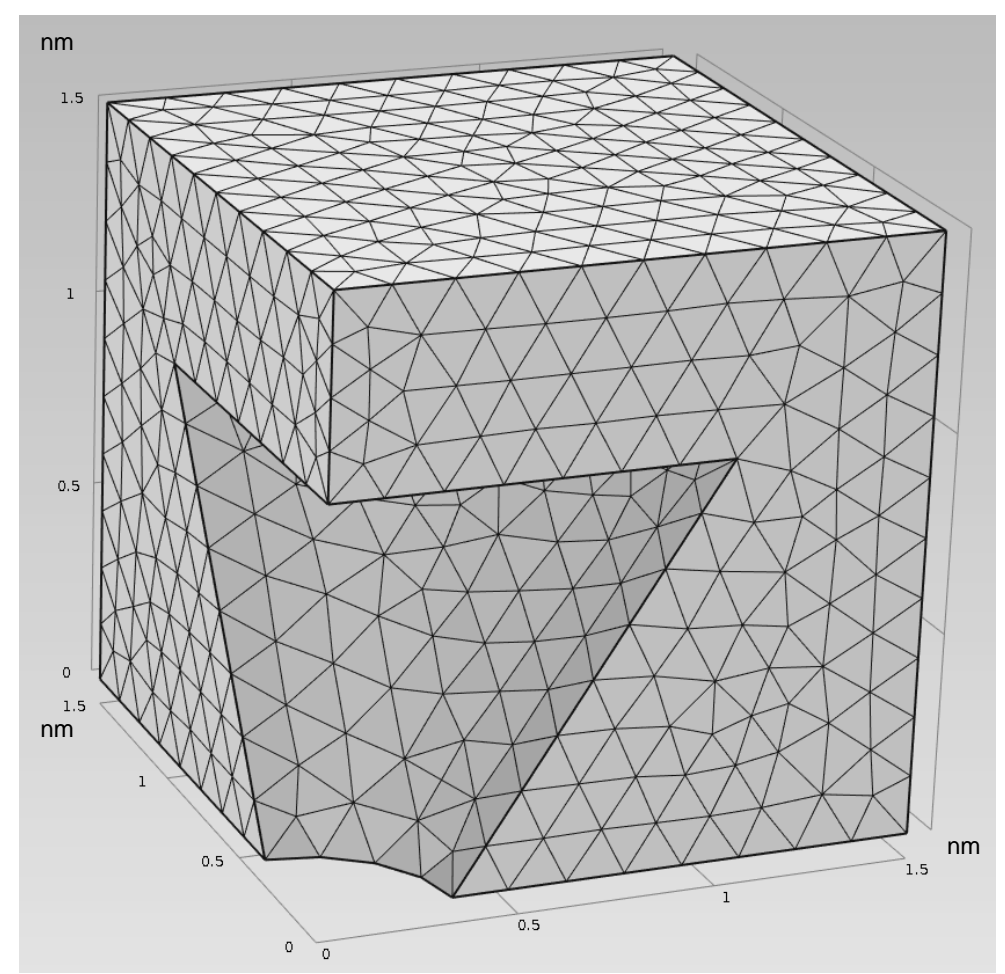

Fig. 5 The flat-tipped cone FEA model used to determine the model for a flat-tipped cone effective cavity in cubic matrix. The finite element mesh is indicated.

For the cubic amorphous matrix, the material properties found in the AFEM simulations in the accompanying paper (Gleadall et al.) are used. Young's modulus is $37.1 \mathrm{GPa}$ and Poisson's ratio is 0.255 . Twelve simulations are conducted in total, in which the side length 
of the cube varies from the same height as the flat-tipped cone to twenty times longer. Further refinement of the mesh has little impact on the results. Young's modulus is calculated by finding the average stress that is applied to the top face of the cube in order to achieve $2 \%$ strain through static linear analysis. The results for normalised Young's modulus versus the volume fraction of effective cavities for all twelve simulations are given in Fig. 6 . The values of Young's modulus found in the simulations are normalised by the initial value for a full cube without any effective cavity. A line of best fit indicates the relationship of

$\bar{E}=e^{-2.483 X e c}$

in which $\bar{E}$ is normalised Young's modulus (no units) and $X_{e c}$ is the volume fraction of the effective cavity in the polymer matrix (no units). The final data point in Fig. 6 is not included when fitting the line of best fit in order to determine Eq. 1 because the relationship between normalised Young's modulus and pore volume fraction begins to deviate from the curve for earlier data points. This is due to high interaction between the effective cavity and the cell boundaries at such a high volume fraction. The model therefore must be considered with caution for Young's modulus drops greater than $50 \%$.

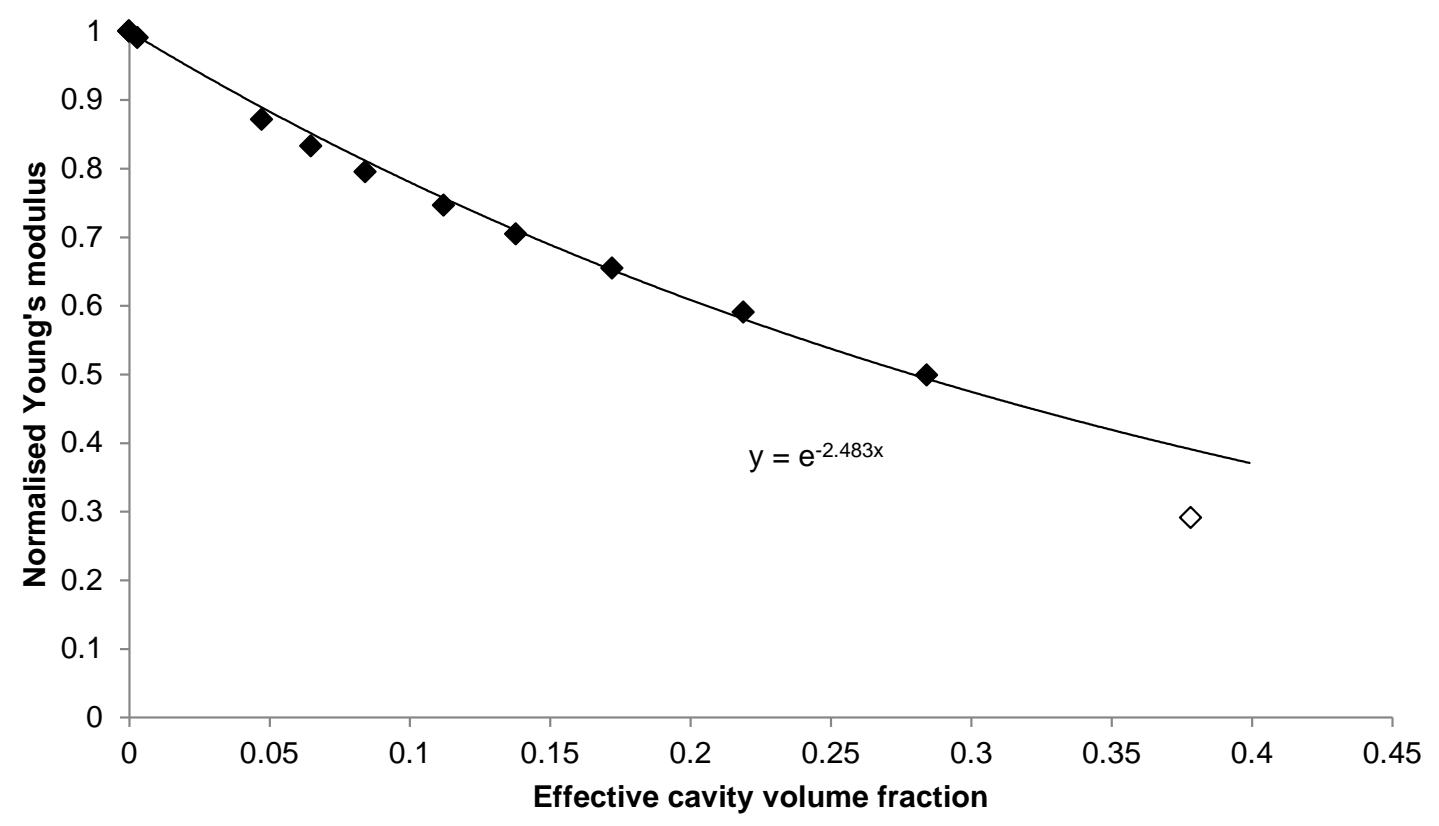

Fig. 6 Normalised Young's modulus versus effective cavity volume fraction for 12 simulations of a flat-tipped cone effective cavity in a cubic polymer matrix. An exponential line of best fitting to the first 11 data points (solid filled diamonds) is shown.

The cone shapes in Fig. 4 show some variation. The value of the exponent in Eq. 1 is dependent on the cone dimensions. In order to test the effect of cone dimensions on the 
relationship found in Eq. 1, the simulations discussed above are repeated for a range of cone dimensions. The fitting shown in Fig. 6 is repeated for each simulation setup. Table 2 gives the cone dimensions and the corresponding value of the exponent for Eq. 1. When fitting the Effective Cavity Theory to experimental data in Section 5.4, error margins indicate the range of Effective Cavity Theory results that the range of exponents in Table 2 gives. For the cone with a height of $2 \mathrm{~nm}$, the unit cell is adjusted to be twice as tall as it is wide to maintain proportionality.

Table 2 Table of cone dimensions used in simulations and respective exponents for Eq. 1.

\begin{tabular}{llll}
\hline Cone height $(\mathrm{nm})$ & Cone large radius $(\mathrm{nm})$ & Cone small radius $(\mathrm{nm})$ & Eq. 1 exponent value \\
\hline 1 & 1 & 0 & -3.439 \\
1 & 1 & 0.333 & -2.483 \\
1 & 1 & 0.667 & -1.845 \\
2 & 1 & 0.333 & -2.041 \\
\hline
\end{tabular}

\subsection{Effect of crystallinity on Young's modulus}

A similar approach is used to develop the model for crystallinity as was used for chain scissions in the previous section. The setup of the model is the same except the flat-tipped cone shaped void is replaced by a cube of material that represents a crystallite particle in an amorphous polymer matrix. Fig. 7 shows the model for a crystal with side lengths equal to $50 \%$ of the overall cube side length including the FEA mesh. As with the chain scission model, symmetry boundary conditions are applied to the left, front and bottom faces to represent an overall cube of eight times the volume of the one shown, which contains a single centrally located crystal particle. The bottom face is restrained in the $z$ direction and the top face is displaced by $2 \%$ of the overall side-length. Poisson's contraction is permitted but the boundaries are constrained to be planar. For the amorphous matrix, Young's modulus is 37.1 GPa and Poisson's ratio is 0.255 , which are the values found in the atomic simulations for amorphous poly(lactide) in the first study in this series (Gleadall et al.). For the crystal particle, Young's modulus is $77.4 \mathrm{GPa}$, which is the mean of the $x, y$ and $z$ values for crystalline poly(lactide) Young's modulus that is found in the atomic simulations in the accompanying paper (Gleadall et al.). Although the values for Young's modulus are higher than expected experimentally, which is discussed in the previous chapter, they are used because no experimental values for crystalline Young's modulus are available. In the simulations here, the absolute values do not affect the results; it is the ratio of crystalline:amorphous Young's moduli that is important. The ratio of 2.1 used here is reasonable. And this ratio is varied later in this section to ensure the results are not critically 
sensitive to it. The shape and isentropic properties of crystals are not well understood so it the model in this thesis is simplified to be isotropic and cube shaped. Poisson's ratio of the crystal particle is 0.239 , which is the mean of the six Poisson's ratio values, $v_{x y}, v_{x z}, v_{y x}, v_{y z}$, $v_{z x}, v_{z y}$, found in the accompanying paper (Gleadall et al.).

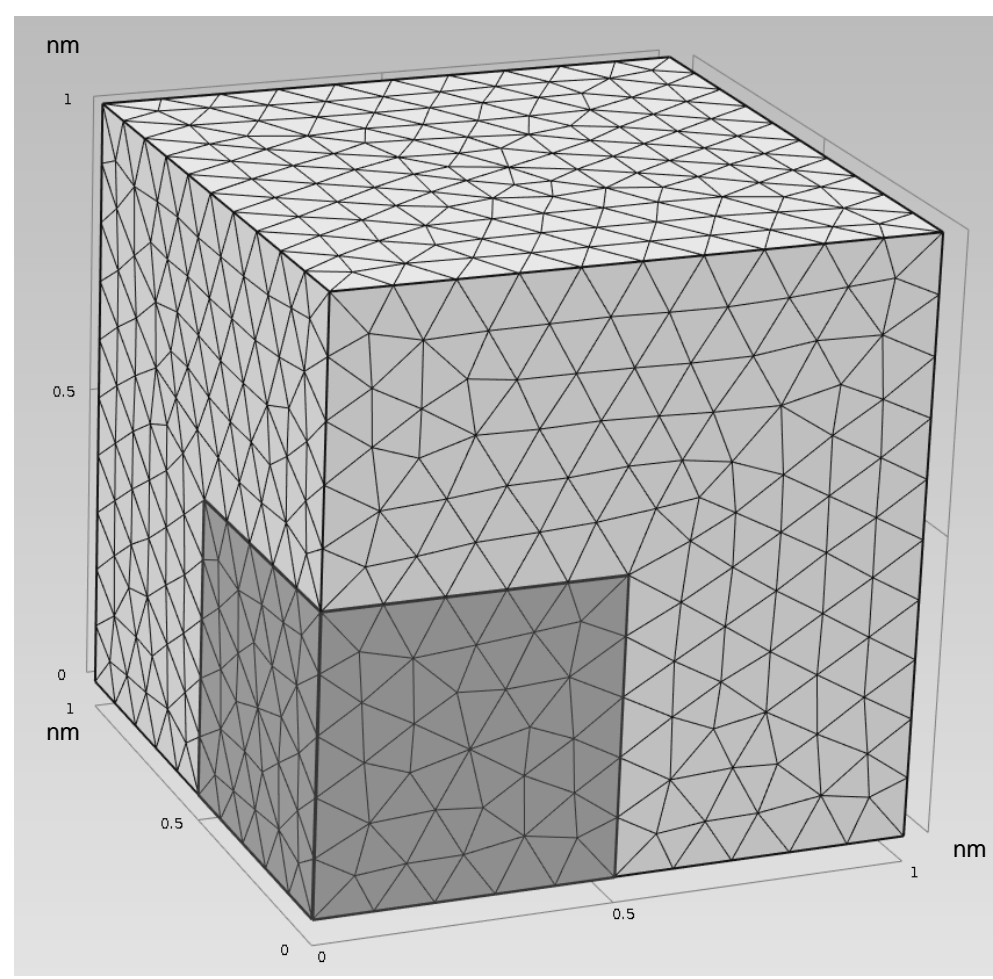

Fig. 7 The crystal cube within a cube FEA model used to determine the model for a crystal particle in an amorphous polymer matrix. The finite element mesh is indicated.

In the FEA simulations used to develop the model for crystallinity, the crystallite particle size remains constant while the size of the cubic cell amorphous matrix varies. As the overall cube size reduces relative to the crystal particle size, the cubic cell represents a polymer in which the crystallites are closer together and occupy a greater volume fraction. Therefore crystallinity is greater. Simulations were completed for 10 different ratios of particle side length to overall cube side length, varying from 0 to 0.9 . The results for normalised Young's modulus versus crystal volume fraction are shown in Fig. 8. The exponential line of best fit indicates that normalised Young's modulus is related to crystal volume fraction $X_{c}$ (no units) by

$\bar{E}=e^{0.754 X c}$.

The value of the exponent in Eq. 2 depends on the ratio between Young's modulus of the crystal particle and amorphous matrix. It also depends of the ratio between the values of 
Poisson's ratio. To determine the effects that the choices of Young's modulus and Poisson's ratio have on the exponent, a range of values for Young's modulus and Poisson's ratio are investigated here. In the above results, the isotropic crystal particle material properties are taken as the mean of the $\mathrm{x}, \mathrm{y}$ and $\mathrm{z}$ uniaxial isentropic material properties found in the atomic simulations of the first paper in this series (Gleadall et al.). Two additional simulations are conducted here, which represent upper and lower bounds. The material properties used are given in Table 3. For the upper bound, the crystal particle Young's modulus is taken as the maximum of the $x, y$ and $z$ Young's modulus and the Poisson's ratio is taken as the maximum of all six Poisson's ratio terms, found in the accompanying paper for crystalline poly(lactide) (Gleadall et al.). This represents a crystal particle with high stiffness oriented in the direction of applied strain. For the lower bound, the crystal particle is given the minimum of all Young's modulus and Poisson's ratio values found in the atomic analysis (Gleadall et al.), which represents a crystal particle with high stiffness oriented normal to the direction of applied strain. The fitting shown in Fig. 8 is repeated for the upper and lower bound setups to find the updated exponent values for Eq. 2. These are given in Table 3 and are used in Section 5.4, when fitting the Effective Cavity Theory to experimental data, in order to indicate error margins.

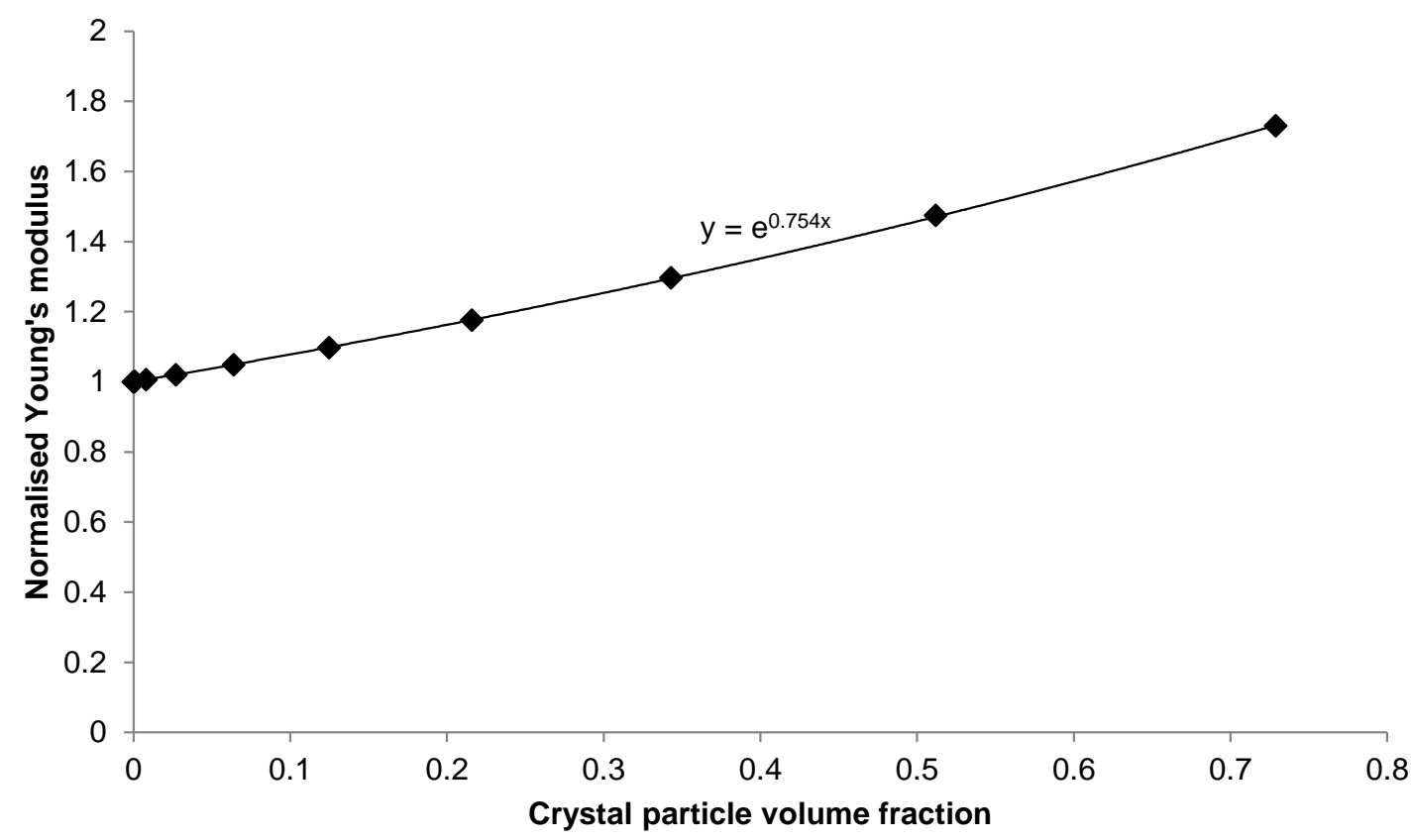

Fig. 8 Normalised Young's modulus versus crystal particle volume fraction for 10 simulations of a crystal particle in a cubic amorphous matrix. An exponential line of best fitting is shown. 
Table 3 Table of crystal material properties used in the simulations and the respective exponents for Eq. 2.

\begin{tabular}{lll|l|ll}
\hline Crystal properties & \multicolumn{2}{l|}{ Young's modulus (GPa) } & \multicolumn{2}{c}{ Poisson's ratio } & \multirow{2}{*}{ Eq. 2 exponent value } \\
\cline { 2 - 5 } & Matrix & Crystal & Matrix & Crystal & \\
\hline Mean $x, y, z$ & 37.1 & 77.4 & 0.255 & 0.239 & 0.754 \\
Lower bound & 37.1 & 128 & 0.255 & 0.328 & 1.246 \\
Upper bound & 37.1 & 50.4 & 0.255 & 0.110 & 0.326 \\
\hline
\end{tabular}

\subsection{Young's modulus of semi-crystalline polymers}

The model for chain scission and the model for crystallinity are combined to consider semicrystalline polymers. Fig. 9 shows how effective cavities are assumed to incorporate both crystal particles and the amorphous polymer matrix. It is out of scope in this work to consider interaction between crystallites and effective cavities. Since the size of crystallites is not known, the model was also successfully fitted to experimental data utilising an assumption of large crystallites such that the voids occupy the amorphous phase only. Future studies may extend the model to consider the interaction between crystallites and effective cavities.

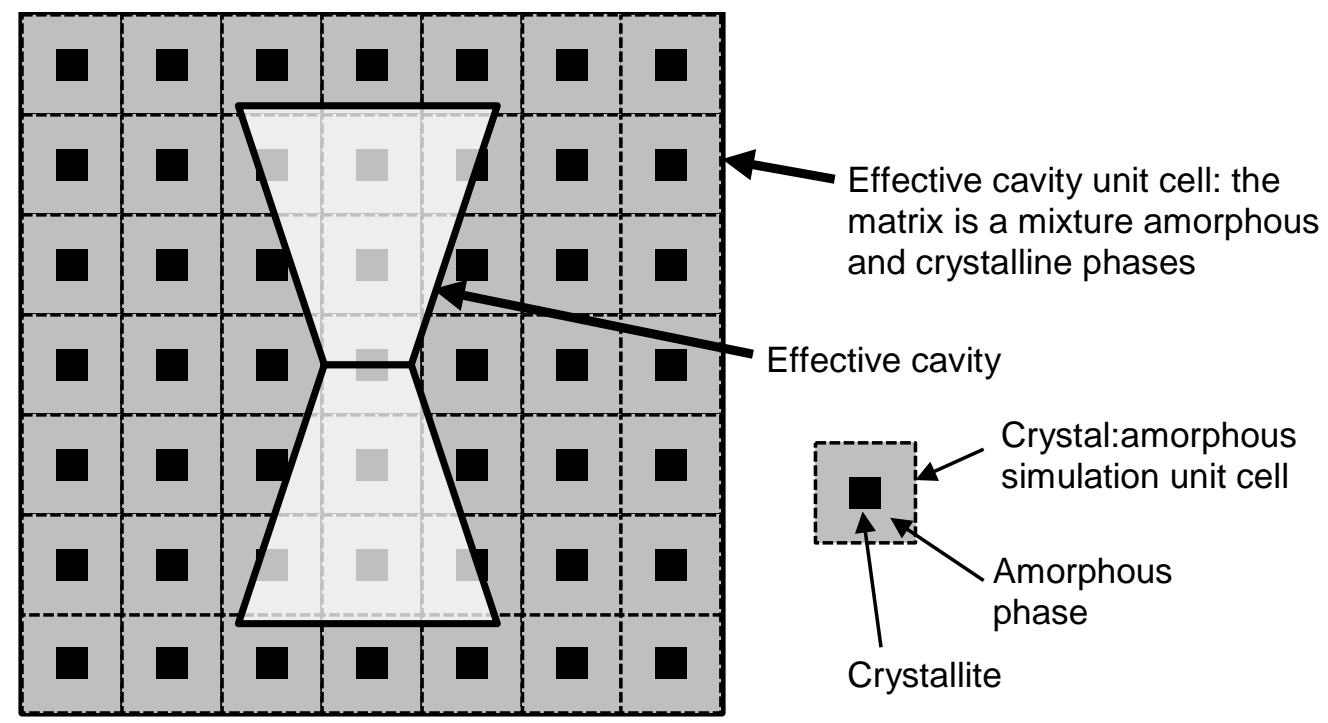

Fig. 9 Effective cavities are considered to occupy a volume of combined crystal and amorphous phases. 
According to Eq. 2, the Young's modulus of the combined crystal particles and amorphous matrix phases, $E_{c}(\mathrm{GPa})$, is empirically assumed to follow the relationship

$$
E_{c}=E_{\infty} e^{0.754 X c}
$$

in which the Young's modulus of a fully amorphous polymer with no effective cavities or crystallinity, $E_{\infty}(\mathrm{GPa})$, is calculated from initial experimental measurements for Young's modulus, number average molecular weight and crystallinity. $E_{c}$ is used as the Young's modulus of the matrix phase in which the effective cavities occur. Therefore for the Effective Cavity Theory, Eq. 1 is used to model the overall polymer Young's modulus, $E_{p}(\mathrm{GPa})$, according to

$$
E_{p}=E_{c} e^{-2.483 X e c} .
$$

Effective cavities are assumed to occur at the sites of initial chain ends as well as the polymer chain ends that result from chain scission to reflect the theory that the effective cavities are associated with chain ends. At any time during degradation the molar number of chains per unit volume, $N_{\text {chain }}\left(\mathrm{mol} \mathrm{m}^{-3}\right)$, can be calculated from molecular weight according to

$$
N_{\text {chain }}=\frac{\rho_{p}}{M_{n}}
$$

in which $\rho_{p}\left(\mathrm{~g} \mathrm{~m}^{-3}\right)$ is the density of the polymer and $M_{n}\left(\mathrm{~g} \mathrm{~mol}^{-1}\right)$ is the number averaged molecular weight. The concentration of chain ends, $C_{\text {end }}\left(\mathrm{mol} \mathrm{m}^{-3}\right)$, is

$$
C_{\text {end }}=2 N_{\text {chain }}
$$

And the volume fraction of effective cavities $X_{\mathrm{ec}}$ (no units) is therefore given by

$$
X_{e c}=C_{e n d} V_{e c}
$$

in which $V_{e c}\left(\mathrm{~m}^{3} \mathrm{~mol}^{-1}\right)$ is the molar volume of each effective cavity.

\subsection{Fitting of the Effective Cavity Theory with experimental data}

In order to demonstrate the Effective Cavity Theory in Eqs. 3 - 7, a fitting of the model to experimental data for the degradation of Young's modulus is completed for five sets of experimental data. These sets of data are chosen because they concern poly(lactide) and have regular measurements for crystallinity, molecular weight and Young's modulus. The only variable that is adjusted in the experimental data fittings is the effective cavity volume $V_{e c}$. The value of $X_{c}$ is measured experimentally in all cases. The density, $\rho_{p}$, is taken to be $1.25 \times 10^{6} \mathrm{~g} \mathrm{~m}^{-3}$. The values of $N_{\text {chain }}$ and $C_{\text {end }}$ are derived from measured $M_{n}$, and therefore 
allow the value of initial $X_{e c}$ to be calculated in Eq. 7 based on the set value of $V_{e c}$. The value of $E_{\infty}$ is derived from Eq. 3 by substitution of the initial measured Young's modulus into Eq. 4 as $E_{p}$, along with the measured and derived values of $X_{c}$ and $X_{e c}$. The experimental setup and initial measurements are given for the five datasets in Table 4, where PBS indicates phosphate buffer solution.

Table 4 Experimental setups and initial measurements for the five sets of data that are used in the Effective Cavity Theory fitting.

\begin{tabular}{|c|c|c|c|c|c|}
\hline Data Set [reference] & $A$ & $B$ & C & $\mathrm{D}$ & $\mathrm{E}$ \\
\hline Initial $M_{n}\left(\mathrm{~g} \mathrm{~mol}^{-1}\right)$ & 159000 & 584000 & 153000 & 152000 & 42000 \\
\hline Initial $X_{c}$ & 0.448 & 0.540 & 0.480 & 0 & 0.570 \\
\hline $\begin{array}{l}\text { Initial Young's } \\
\text { modulus (GPa) }\end{array}$ & 0.668 & 0.100 & 6.86 & 5.58 & 1.43 \\
\hline Sample type & $\begin{array}{l}0.8 \mathrm{~mm} \\
\text { plate }\end{array}$ & $\begin{array}{l}0.050 \mathrm{~mm} \\
\text { film }\end{array}$ & $2 \mathrm{~mm}$ rod & $3 \mathrm{~mm}$ rod & $\begin{array}{l}0.033 \mathrm{~mm} \\
\text { film }\end{array}$ \\
\hline Polymer type & PLLA & PLLA & PLLA & PLLA & PLLA \\
\hline Degradation medium & $\begin{array}{l}37^{\circ} \mathrm{C} \text { PBS } \\
\mathrm{pH} 7.4\end{array}$ & $\begin{array}{l}37^{\circ} \mathrm{C} \text { PBS } \\
\mathrm{pH} 7.4\end{array}$ & $\begin{array}{l}38^{\circ} \mathrm{C} \text { PBS } \\
\mathrm{pH} 7.4\end{array}$ & $\begin{array}{l}38^{\circ} \mathrm{C} \text { PBS } \\
\mathrm{pH} 7.4\end{array}$ & $\begin{array}{l}37^{\circ} \mathrm{C} \text { PBS } \\
\mathrm{pH} 7.4\end{array}$ \\
\hline
\end{tabular}

Fig. 10 shows the experimental data for Young's modulus along with the Effective Cavity Theory results. In Fig. 10 (c) and (d), crystallinity was not measured at week 12 so the values used for the Effective Cavity Theory fitting are taken as the average of the measurements at weeks 8 and 16. The Effective Cavity Theory fitting gives discrete data points because Eqs. 4 - 7 are used at each experimental measurement time. A molar volume of $9.41 \times 10^{-3} \mathrm{~m}^{3} \mathrm{~mol}^{-1}$ is used for effective cavity volume $V_{e c}$ for all five data sets in Fig. 10. It is justifiable to use several effective cavity volumes, since the samples may have different polymer chain configuration due to the different experimental setups. However, the value is not adjusted between fittings in order to reduce the number of parameters that can be varied to achieve the best fitting. The fittings can be improved if the value of $V_{e c}$ is allowed to vary. The error bars indicate the minimum and maximum Young's moduli found by the Effective Cavity Theory for all combinations of exponents given in Table 2 and Table 3 being used in Eq. 4 and Eq. 3, respectively. The Effective Cavity Theory gives a very good fitting to the experimental data.

The model is able to consider Young's modulus increase due to crystallinity as demonstrated in the first two data points in Fig. 10 (c) and the first few weeks in Fig. 10 (d). The Effective Cavity Theory also correctly reproduces the slightly erratic nature of Young's modulus 
reduction Fig. 10 (a). The trend can be linked to the molecular weight experimental measurements, which fluctuate in a similar manner to Young's modulus. The Effective Cavity Theory is intended for Young's modulus reductions up to $50 \%$ as discussed in relation to Fig. 6. Therefore, for the later stages in each of the fittings, the results should be considered with caution. However, the data fittings quite accurately model Young's modulus reduction to zero. For the chosen effective cavity volume, the experimental data considered in Fig. 10 (e) has a very high initial volume fraction of effective cavities (56\%) compared to the other four studies (4-15\%) because it has a much lower initial molecular weight. Although the Effective Cavity Theory is not intended for the data in Fig. 10 (e), it can be seen to achieve quite a good fitting, using the same volume of effective cavity.

The simple model presented in Eqs. 3 - 7 for the Effective Cavity Theory is based on the model for an isolated flat-tipped cone void and does not consider overlap of the effective cavities. If effective cavity overlap was considered in the Effective Cavity Theory, following a theory such 

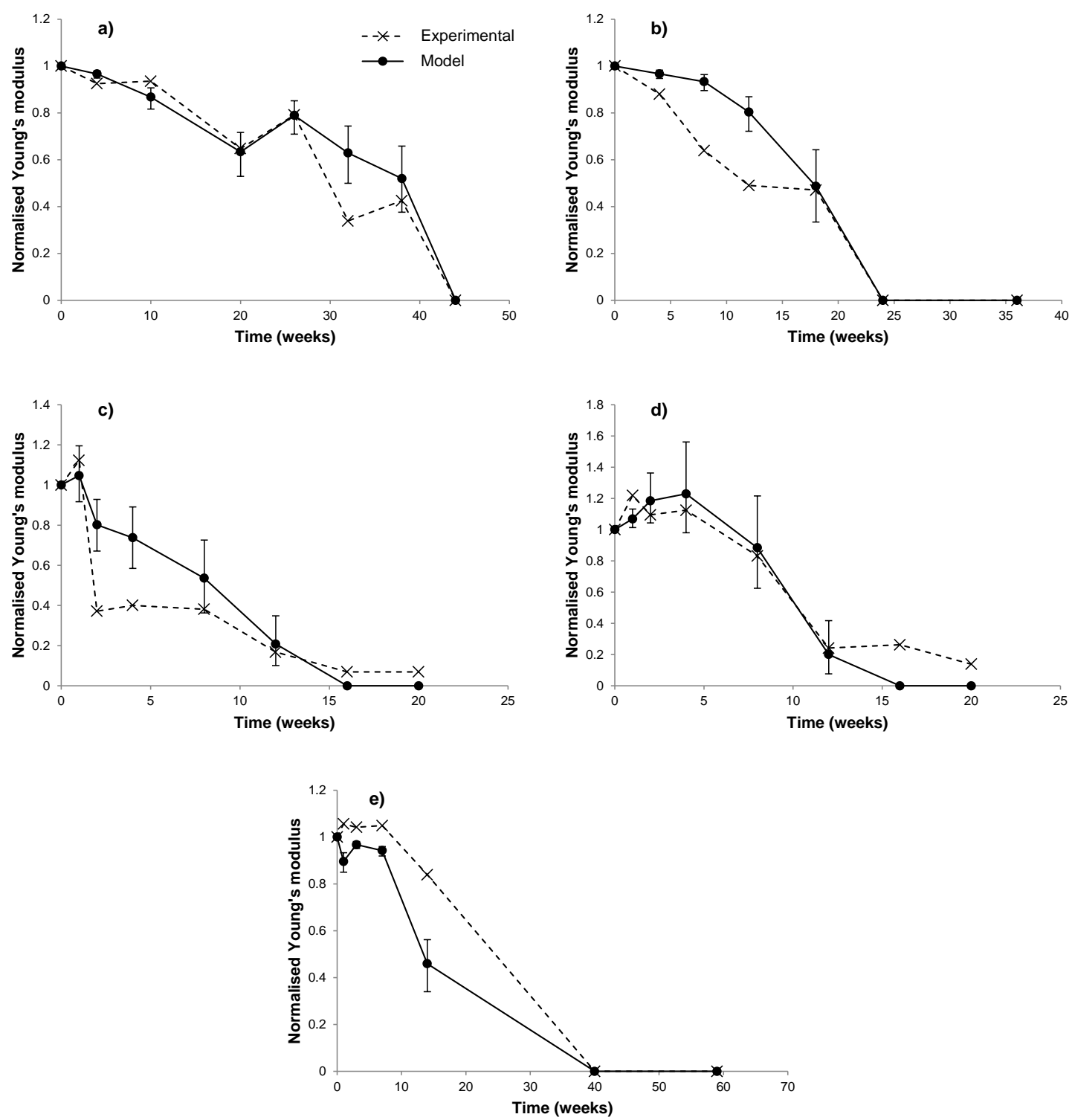

Fig. 10 Experimental data and the Effective Cavity Theory model for Young's modulus in five degradation studies: a) , b) , c) and d) and e).

as crystal overlap based on Avrami Theory presented by Han and Pan (Han and Pan, 2009), the rate of Young's modulus reduction would reduce as it approached zero. Such a trend may result in a better fitting towards the end of degradation for the experimental data sets in Fig. 10 (c) and (d). The value of $V_{e c}$ used in the Effective Cavity Theory model represents a cone height of $2.74 \mathrm{~nm}$ for the effective cavities. In the AFEM analysis, the chain scissions affect a smaller volume than that used in the fitting here. This can be explained by the fact that the AFEM analysis underestimates the effect of chain scission due to polymer properties being simulated at 0 Kelvin.

The Effective Cavity Theory may be combined with existing mathematical models from the literature for biodegradable polymer degradation, such as those presented in earlier work 
(Gleadall et al., 2012; Han and Pan, 2009; Han et al., 2010), to give a combined model for molecular weight, crystallinity and Young's modulus throughout degradation.

\section{Conclusion}

The atomic finite element method, developed in the accompanying paper (Gleadall et al.), was used to analyse the effect of chain scission on Young's modulus degradation in biodegradable polymers. Up to 258 scissions were applied to the amorphous polymer structure in order to model an overall trend for Young's modulus versus molecular weight in a degrading poly(lactide). It was found that Young's modulus degradation delayed behind molecular weight reduction. This trend has also been found in many experimental studies in the literature.

The effect of individual chain scissions was also studied. It was found that chain scissions may instantiate a volume of reduced-stiffness polymer near to the scission. The Effective Cavity Theory was presented, in which effective cavities are considered to exist by chain scissions. In addition to modelling Young's modulus degradation due to chain scission, the Effective Cavity Theory also considers the effect of crystallinity increases during degradation. Finite element analysis simulations were conducted to find a relationship between (i) the volume fraction of effective cavities and Young's modulus, and (ii) crystallinity and Young's modulus. The Effective Cavity Theory model was fit to experimental data for Young's modulus in five sets of data in the literature. A good fitting was achieved when the same model parameter values were used for all five data fittings; the only inputs to the model that varied were the experimentally measured values of molecular weight, crystallinity and initial Young's modulus.

\section{Acknowledgements}

Andrew Gleadall acknowledges an EPSRC PhD studentship and a partial PhD studentship from the University of Leicester.

The authors would also like to acknowledge James McAliley for supplying the molecular structure coordinate files and PLAFF2 force field files with his thesis (McAliley, 2009).

\section{References}


Ding, L., Davidchack, R., Pan, J., 2011. A molecular dynamics study of Young's modulus change of semi-crystalline polymers during degradation by chain scissions. Journal of the Mechanical Behavior of Biomedical Materials 5, 224-230.

Duek, E.A.R., Zavaglia, C.A.C., Belangero, W.D., 1999. In vitro study of poly(lactic acid) pin degradation. Polymer 40, 6465-6473.

Gleadall, A., Pan, J., Atkinson, H., 2012. A simplified theory of crystallisation induced by polymer chain scissions for biodegradable polyesters. Polymer Degradation and Stability 97 , 1616-1620.

Gleadall, A., Pan, J., Ding, L., Kruft, M.-A., Curco, D., An atomic finite element model for biodegradable polymers. Part 1. Formulation of the finite elements. Journal of the Mechanical Behavior of Biomedical Materials.

Han, X., Pan, J., 2009. A model for simultaneous crystallisation and biodegradation of biodegradable polymers. Biomaterials 30, 423-430.

Han, X., Pan, J., Buchanan, F., Weir, N., Farrar, D., 2010. Analysis of degradation data of poly(I-lactide-co-I,d-lactide) and poly(I-lactide) obtained at elevated and physiological temperatures using mathematical models. Acta Biomaterialia 6, 3882-3889.

Lam, K.H., Nieuwenhuis, P., Molenaar, I., Esselbrugge, H., Feijen, J., Dijkstra, P.J., Schakenraad, J.M., 1994. Biodegradation of porous versus non-porous poly(L-lactic acid) films. Journal of Materials Science: Materials in Medicine 5, 181-189.

McAliley, J.H., 2009. Development of improved torsional potentials in classical force field descriptions of poly (lactic acid), Department of Chemical and Biomolecular Engineering. Clemson University, United States.

Migliaresi, C., Fambri, L., Cohn, D., 1994. A study on the in vitro degradation of poly(lactic acid). Journal of Biomaterials Science, Polymer Edition 5, 591-606.

Saha, S.K., Tsuji, H., 2006. Effects of rapid crystallization on hydrolytic degradation and mechanical properties of poly(I-lactide-co- $\varepsilon$-caprolactone). Reactive and Functional Polymers 66, 1362-1372.

Tsuji, H., 2000. In vitro hydrolysis of blends from enantiomeric poly(lactide)s. Part 1. Wellstereo-complexed blend and non-blended films. Polymer 41, 3621-3630.

Tsuji, H., 2002. Autocatalytic hydrolysis of amorphous-made polylactides: effects of L-lactide content, tacticity, and enantiomeric polymer blending. Polymer 43, 1789-1796.

Tsuji, H., 2003. In vitro hydrolysis of blends from enantiomeric poly(lactide)s. Part 4: wellhomo-crystallized blend and nonblended films. Biomaterials 24, 537-547.

Tsuji, H., Ikada, Y., 1995. Properties and morphologies of poly(L-lactide): 1. Annealing condition effects on properties and morphologies of poly(L-lactide). Polymer 36, 2709-2716. 
Tsuji, H., Mizuno, A., Ikada, Y., 2000. Properties and morphology of poly(L-lactide). III. Effects of initial crystallinity on long-term in vitro hydrolysis of high molecular weight poly(Llactide) film in phosphate-buffered solution. Journal of Applied Polymer Science 77, 14521464.

Tsuji, H., Muramatsu, H., 2001. Blends of aliphatic polyesters: V non-enzymatic and enzymatic hydrolysis of blends from hydrophobic poly(l-lactide) and hydrophilic poly(vinyl alcohol). Polymer Degradation and Stability 71, 403-413.

Tsuji, H., Suzuyoshi, K., 2002a. Environmental degradation of biodegradable polyesters 1. Poly([var epsilon]-caprolactone), poly[(R)-3-hydroxybutyrate], and poly(L-lactide) films in controlled static seawater. Polymer Degradation and Stability 75, 347-355.

Tsuji, H., Suzuyoshi, K., 2002b. Environmental degradation of biodegradable polyesters 2. Poly([var epsilon]-caprolactone), poly[(R)-3-hydroxybutyrate], and poly(L-lactide) films in natural dynamic seawater. Polymer Degradation and Stability 75, 357-365.

Wang, Y., Han, X., Pan, J., Sinka, C., 2010. An entropy spring model for the Young's modulus change of biodegradable polymers during biodegradation. Journal of the Mechanical Behavior of Biomedical Materials 3, 14-21.

Weir, N., Buchanan, F., Orr, J., Dickson, G., 2004. Degradation of poly-L-lactide. Part 1: in vitro and in vivo physiological temperature degradation. Proceedings of the Institution of Mechanical Engineers, Part H: Journal of Engineering in Medicine 218, 307-319.

Yuan, X., Mak, A.F.T., Yao, K., 2002. In vitro degradation of poly(L- lactic acid) fibers in phosphate buffered saline. Journal of Applied Polymer Science 85, 936-943. 